\title{
Modeling Thermal Expansion of $\mathrm{Ni}_{2} \mathrm{MnGe}$
}

\author{
M. PugacZowa-Michalska \\ Institute of Molecular Physics, Polish Academy of Sciences \\ M. Smoluchowskiego 17, 60-179 Poznań, Poland
}

\begin{abstract}
The present study of $\mathrm{Ni}_{2} \mathrm{MnGe}$ is focused on describing the thermal properties of the alloy in the framework of first-principles electronic structure calculations coupled with a Debye treatment of the vibrating lattice. The electronic structure of $\mathrm{Ni}_{2} \mathrm{MnGe}$ has been studied using the full-potential nonorthogonal local-orbital minimum basis method. Two approximations for Grüneisen parameter $\gamma$, i.e. Slater's and Dugdale and MacDonald's expressions were assumed.
\end{abstract}

PACS numbers: 71.20.Lp, 71.20.Be, 65.40.De

\section{Introduction}

Interest in the Heusler alloys arises from the fact that they are considered to be systems for spintronic and magnetically driven actuators. Characteristic properties of $\mathrm{Ni}_{2} \mathrm{MnGe}$ as a film attempted to recognize in the previous experimental studies [1-5]. The structural, magnetic and magneto-optical properties of $\mathrm{Ni}_{2} \mathrm{MnGe}$ film prepared by flash evaporation have been measured in [1]. Anomalous magnetotransport properties of epitaxial $\mathrm{Ni}_{2} \mathrm{MnGe}$ film grown by molecular beam epitaxy have been reported in [4]. First principles band structure calculations for bulk $\mathrm{Ni}_{2} \mathrm{MnGe}$ in $L 2_{1}$ structure have shown spin dependent hybridization of $3 d$ states of Mn atom with electronic states of Ni-3d and Ge-5sp [6]. The large exchange splitting of the $3 \mathrm{~d}$ states of $\mathrm{Mn}$ results in the large value of the spin moment of $\mathrm{Mn}$ atom $\left(3.37 \mu_{\mathrm{B}}\right)$ as well as the large value of the total magnetic moment $\left(3.67 \mu_{\mathrm{B}}\right)$ [6]. In ferromagnet/semiconductor heterostructures, in which the studied $\mathrm{Ni}_{2} \mathrm{MnGe}$ Heusler alloy has been recently used $[2,3]$, stresses may be generated at the interfaces due to thermal expansion mismatch. Knowledge of thermal expansion of materials is important in numerous applications. That is the reason to expand earlier theoretical study of $\mathrm{Ni}_{2} \mathrm{MnGe}$ on the thermal properties discussion. In order to make a prediction of the thermodynamic properties of $\mathrm{Ni}_{2} \mathrm{MnGe}$ the Debye-Grüneisen model was chosen to account for the vibrational contribution and calculate the coefficient of thermal expansion.

\section{Method of calculation}

We adopted strategy in studying thermal expansion of $\mathrm{Ni}_{2} \mathrm{MnGe}$ proposed by Moruzzi et al. [7]. The first step of the study of $\mathrm{Ni}_{2} \mathrm{MnGe}$ is based on the first principles electronic structure calculations and estimation of parameters of equation of states [6]. Debye temperatures and Grüneisen parameter are derived from an analysis of the compressional characteristics of rigid-lattice binding curves. Next, we used Debye parameter and Grüneisen parameter to define the contribution of the lattice vibrations to the free energy.

According to Moruzzi's scheme [7] the Helmholtz free energy of a system comprises the total energy $E(V)$ from $a b$ initio total energy calculation and the free energy of the vibrating lattice. The Helmholtz free energy can be expressed as

$$
\begin{aligned}
& F(V, T)=E(V)+F^{\mathrm{vib}}(V, T) \\
& \quad=E(V)+E_{D}^{\mathrm{vib}}(V, T)-T S_{D}^{\mathrm{vib}}(V, T),
\end{aligned}
$$

where $E_{D}^{\mathrm{vib}}(V, T)$ and $S_{D}^{\mathrm{vib}}(V, T)$ are the thermal vibrational energy and the vibrational entropy, respectively, at volume $V$ and temperature T. In Eq. (1) no electronic contribution was considered, which would not cause any problem below room temperature. To calculate the Helmholtz energy $F(V, T)$ in Eq. (1) the DebyeGrüneisen model has been employed and the total energy values obtained from the full-potential nonorthogonal local-orbital minimum basis (FPLO) method $[8,9]$ have been used. In the FPLO calculations the exchange-correlation potential in the form of [10] has been applied. The Brillouin zone integrations were performed using the tetrahedron method and $732 k$-points used in its irreducible part. Other details of the total energy calculations are exactly the same as described in [6]. The thermal vibrational energy and the vibrational entropy can be expressed in the terms of the Debye function $D\left(\frac{\Theta_{\mathrm{D}}}{T}\right)$ :

$$
\begin{aligned}
& E_{D}^{\mathrm{vib}}(V, T)=E_{0}+3 k_{\mathrm{B}} T D\left(\frac{\Theta_{\mathrm{D}}}{T}\right), \\
& S_{D}^{\mathrm{vib}}(V, T)=3 k_{\mathrm{B}} \\
& \times\left\{\frac{4}{3} D\left(\frac{\Theta_{\mathrm{D}}}{T}\right)-\ln \left[1-\exp \left(-\frac{\Theta_{\mathrm{D}}}{T}\right)\right]\right\},
\end{aligned}
$$

where $\Theta_{\mathrm{D}}$ is the Debye temperature. The Debye func- 
tion values $D\left(\frac{\Theta_{\mathrm{D}}}{T}\right)$ are listed in tabulated form [11] or calculated by numerical methods. $k_{\mathrm{B}}$ is the Boltzmann constant, $E_{0}$ is the zero-point energy

$$
E_{0}=\frac{9}{8} k_{\mathrm{B}} \Theta_{\mathrm{D}} \text {. }
$$

The Debye function is given by

$$
D(y)=\frac{3}{y^{3}} \int_{0}^{y} \frac{x^{3}}{\mathrm{e}^{x}-1} \mathrm{~d} x .
$$

Anharmonic effects in the vibrating lattice are modelled by a single Grüneisen parameter $\gamma$. The definition of the Grüneisen parameter is

$$
\gamma=-\frac{\partial \ln \Theta_{\mathrm{D}}}{\partial \ln V}
$$

In Eq. (1) as well as in (2) and (3) the volume dependence enters by way of $\Theta_{\mathrm{D}}$ through the relation

$$
\Theta_{\mathrm{D}}=\Theta_{D, 0}\left(\frac{V_{0}}{V}\right)^{\gamma},
$$

where $\Theta_{\mathrm{D}, 0}$ is the Debye temperature at equilibrium volume $V_{0}$. The Grüneisen parameter can be calculated according to two well-known approximations [12, 14]. These approximations can be combined using the following expression similar to written one in [15]:

$$
\gamma(V)=\frac{l-1}{3}-\frac{V}{2} \frac{\partial^{2}\left(p V^{(2 l / 3)}\right) / \partial V^{2}}{\partial\left(p V^{(2 l / 3)}\right) / \partial V},
$$

where $p(V)=-\partial E(V) / \partial V$. When $l=0$, one immediately obtains the Slater approximation [13], which considers the material as an elastic medium and assumes the independence of Poisson's ratio on volume. The following choice of $l=1$ fulfills Dugdale and MacDonald approximation [14]. Thus, Eq. (8) will be reduced to the expression proposed by Dugdale and MacDonald [14], which follows from the assumption that in cubic crystal all the force constants have the same volume dependence. Thus the volume dependence of the Debye temperatures have been evaluated from Eq. (7) using the above-mentioned approximations. Next, the free energy of vibrating system can be calculated as a function of $V$ and $T$. Minimization of the free energy as a function of volume at some values of temperature allowed us to determine the theoretical volume-temperature dependence.

\section{Results and discussion}

In the presented calculations the Debye temperature at equilibrium volume $\Theta_{D, 0}$ was assumed the value estimated in [6] (about $322 \mathrm{~K}$ ). Figure 1 presents the temperature dependence of reduced atomic volume $\left(V / V_{0}\right)$ of $\mathrm{Ni}_{2} \mathrm{MnGe}$ for abovementioned approximations of $\gamma[13,14]$. The calculated thermal expansion reproduces all known feature: increase in cubic $\mathrm{Ni}_{2} \mathrm{MnGe}$ with temperature. The calculated dependence show that the studied $\mathrm{Ni}_{2} \mathrm{MnGe}$ expands with heat, somewhat larger for the Slater approximation than for Dugdale and MacDonald approximation of $\gamma$. According to the Slater approximation the expansion of $\mathrm{Ni}_{2} \mathrm{MnGe}$ due to heat, i.e. from $10 \mathrm{~K}$ to $300 \mathrm{~K}$ is about $0.28 \%$. However, the same increase in the temperature of sample cause the

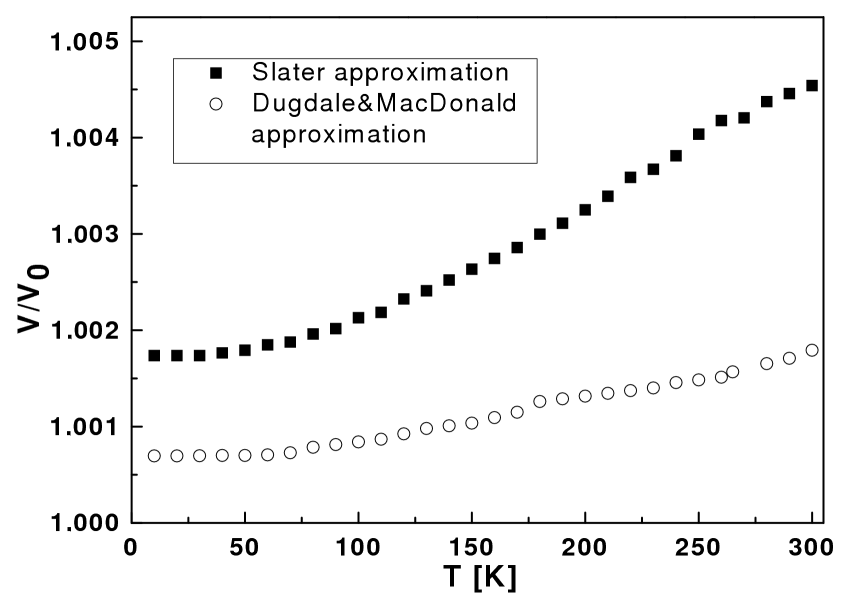

Fig. 1. Calculated thermal expansion of $\mathrm{Ni}_{2} \mathrm{MnGe}$. The squares present the calculated results with respect to the Slater approximation for $\gamma$. The circles are calculated values according to Dugdale and MacDonald approximation for $\gamma . V_{0}$ is an equilibrium volume from $a b$ initio calculations [6].

expansion of $\mathrm{Ni}_{2} \mathrm{MnGe}$ of about $0.11 \%$ for Dugdale and MacDonald approximation of $\gamma$. Thermal expansion is characterized by coefficients of volume and linear expansion. These coefficients are determined by the relative change in volume or length of the system related to $1 \mathrm{~K}$. For cubic materials the relation between the coefficient of volume expansion $\alpha_{V}(V)$ and the coefficient of linear expansion $\alpha_{l}(V)$ can be expressed in a simple form: $\alpha_{V}(V) \simeq 3 \alpha_{l}(V)$. In this work, the above relation is fulfilled because of the studied $\mathrm{Ni}_{2} \mathrm{MnGe}$ having cubic structure $\left(L 2_{1}\right)$. When the Helmholtz free energy is expressed as a function of volume $V$ and temperature $T$, the equilibrium $V(T)$ and thus the coefficient of linear expansion can be calculated through the minimum of $F(V, T): \partial F(V, T) / \partial V=0$. The coefficient of thermal expansion $\alpha$ is

$$
\alpha_{V}(T)=\frac{1}{V_{0}} \frac{\mathrm{d}\left(V_{0}\right)}{\mathrm{d} T} .
$$

The obtained value of volume coefficient of thermal expansion at room-temperature is $\alpha_{V}=8.39 \times 10^{-6} \mathrm{~K}^{-1}$ for Dugdale and MacDonald approximation. The assessment at room temperature for volume coefficient of thermal expansion of $\mathrm{Ni}_{2} \mathrm{MnGe}$ gives value of $\alpha_{V}=8.38 \times 10^{-6} \mathrm{~K}^{-1}$ for the Slater approximation. Our calculations suggest that both theoretical modeling of anharmonic Grüneisen parameter $\gamma$ results in the same volume coefficient of thermal expansion for $\mathrm{Ni}_{2} \mathrm{MnGe}$ at room temperature. It is not a surprising result in the light of study of thermal expansion some of cubic transition metals. For majority of transition cubic metals recently studied in [16] the difference between thermal expansion coefficient obtained using Slater expression of $\gamma$ and Dugdale-MacDonald one is nearly neglected below the room temperature. The experimental investigation of the thermal expansion of 
$\mathrm{Ni}_{2} \mathrm{MnSn}$ and $\mathrm{Ni}_{2} \mathrm{MnSb}$ Heusler alloys [17] has shown that the thermal expansion coefficient has the same order of value. Moreover, one expects that the obtained theoretical value of the thermal expansion coefficient for studied $\mathrm{Ni}_{2} \mathrm{MnGe}$ is somewhat underestimated due to natural limitation of the Debye-Grüneisen model.

\section{References}

[1] V.O. Oksenenko, L.N. Trofimova, Yu.N. Petrov, Y.V. Kudryavtsev, J. Dubowik, Y.P. Lee, J. Appl. Phys. 99, 063902 (2006).

[2] J.W. Dong, J. Lu, J.Q. Xie, L.C. Chen, R.D. James, S. McKernan, C.J. Palmstrøm, Physica E 10, 428 (2001).

[3] J. Lu, J.W. Dong, J.Q. Xie, S. McKernan, C.J. Palmstrøm, Y. Xin, Appl. Phys. Lett. 83, 2393 (2003).

[4] M.S. Lund, J.W. Dong, J. Lu, X.Y. Dong, C.J. Palmstrøm, C. Leighton, Appl. Phys. Lett. $\mathbf{8 0 ,} 4798$ (2002).

[5] R.J. Kim, N.N. Lee, B.J. Kim, Y.P. Lee, Y.V. Kudryavtsev, K.W. Kim, IEEE Trans. Magn. 41, 3443 (2005).

[6] M. Pugaczowa-Michalska, J. Alloys Comp. 427, 54 (2007).
[7] V.L. Moruzzi, J.F. Janak, K. Schwarz, Phys. Rev. B 37, 790 (1988).

[8] K. Koepernik, H. Eschrig, Phys. Rev. B 59, 1743 (1999).

[9] I. Opahle, K. Koepernik, H. Eschrig, Phys. Rev. B 60, 14035 (1999)

[10] J.P. Perdew, Y. Wang, Phys. Rev. B 45, 13244 (1992).

[11] G.T. Furukawa, T.B. Douglas, N. Pearlman, in: American Institute of Physics Handbook, 3rd ed., McGraw-Hill, New York 1972, Sect. 4e, p. 4.

[12] Y. Wang, R. Ahuja, B. Johansson, Int. J. Quant. Chem. 96, 501 (2004).

[13] J.C. Slater, Introduction to Chemical Physics, McGraw-Hill, New York 1939.

[14] J.S. Dugdale, D.K.C. MacDonald, Phys. Rev. 89, 832 (1953).

[15] E. Wasserman, L. Strixrude, R.E. Cohen, Phys. Rev. B 53, 8296 (1996).

[16] X-G. Lu, M. Selleby, B. Sundman, Acta Mater. 53 2259 (2005).

[17] S.M. Podgornych, V.A. Kazancev, E.I. Shreder, Phys. Met. Metalloved. 86, 69 (1998). 